\title{
Thermodynamic analysis of medium pressure reciprocating natural gas expansion engines
}

\author{
Mahmood Farzaneh-Gord ${ }^{1}$, Soheil Izadi ${ }^{1}$, Seyed Iman Pishbin ${ }^{2}$, Hamideh Sheikhani ${ }^{3}$, \\ Mahdi Deymi-Dashtebayaz ${ }^{4 *}$
}

\author{
${ }^{1}$ Shahrood University of Technology, The Faculty of Mechanical Engineering, Shahrood, Iran \\ ${ }^{2}$ Khayyam University of Mashhad, Department of Mechanical Engineering, Mashhad, Iran \\ ${ }^{3}$ National Iranian Gas Company, Research and Development Department, Khorasan Razavi Province \\ ${ }^{4}$ Hakim Sabzevari University, Faculty Members of Mechanical Engineering, Sabzevar, Iran \\ "Corresponding author: e-mail: meh_deimi@yahoo.com
}

\begin{abstract}
Natural gas pressure has to be reduced from medium pressure of $1.724 \mathrm{MPa}(250 \mathrm{psia})$ to lower pressure of $0.414 \mathrm{MPa}(60 \mathrm{psia})$ at Town Border pressure reduction Station (TBS). Currently, the pressure reduction is carried out by throttling valves while considerable amount of pressure energy is wasted. One of the equipment which could be used to recover this waste energy is the reciprocating expansion engine. The purpose of this research is to simulate one-sided reciprocating expansion engine thermodynamically for TBS pressure range. The simulation is based on first law of thermodynamics, conversation of mass and ideal gas assumptions. The model could predict in-cylinder pressure and in-cylinder temperature at various crank angles. In addition, the effects of the engine geometrical characteristics, such as intake and exhaust port area and ports timing on the Indicated work per cycle output are investigated.
\end{abstract}

Keywords: Expansion engine, thermodynamic, first law, optimization, ideal gas model.

\section{INTRODUCTION}

The shortages of energy resources, global warming and environmental problems have motivated the searches for more efficient methods of energy conservation. One of the industries that have high energy dissipation is natural gas pipelines. In Iran (and perhaps in many countries), natural gas is transported through transmission pipelines at high pressures (5-7 MPa) from production locations to consuming points. At consumption points, or when crossing into a lower pressure pipeline, the pressure of the gas must be reduced. This pressure reduction takes place in City Gate Stations (CGSs) and Town Border Stations (TBSs). At CGSs and TBSs, the pressure are reduced from 5-7 $\mathrm{MPa}$ to $1.7 \mathrm{MPa}$ and $1.7 \mathrm{MPa}$ to 0.4 MPa in high-pressure intrastate pipelines respectively. Currently, gas pressure reduction is accomplished by using throttle-valves in all of Iran's CGSs and TBSs, where the constant-enthalpy expansion takes place and a considerable amount of energy is wasted. Different scenarios have suggested for recovering this huge amount of energy.

Much research has been done studying the use of natural gas pressure exergy, focusing on the pressure drop stations. One example is the work of Bisio ${ }^{1}$ that he examined systems to use this exergy, including a mechanical system to compress air. Expansion turbines (turbo expanders) are also available for generating electricity from this exergy. In another study, Pozivill ${ }^{2}$ has studied the use of turbo-expanders in natural gas pressure-drop stations with commercial software, AspenTech's HYSYS process simulator. He investigated the effects of utilizing an expansion machine that was considered to be a turbo expander. Also, Greeff et al. ${ }^{3}$ have studied the integration of turbo-expanders into various high-pressure exothermic chemical-synthesis processes. They demonstrated successful integration of a turbo-expander with meaningful energy savings. Mirandola and Minca ${ }^{4}$ presented several suggestions such as number of expansion steps about design of power generation systems from high pressure gas in different inlet and outlet conditions.

Farzaneh-Gord et al. ${ }^{5-10}$ also offered various scenarios for recovering pressure energy of natural gas stream in pipelines. In one study ${ }^{8}$, based on recorded data and measured conditions of natural-gas at Khangiran pressure drop station for a year, the amount of available work from the station has been calculated. The proposed system were consists of preheating and an expander turbine.

Using reciprocating expansion engine in TBS is one of newest methods that studied in this research. Expansion engines have several advantages over expansion turbines; such as:

1. They can be operated with wet natural gas flow (hydrate in natural gas).

2. Generally expansion engines are less expensive than expansion turbines especially for capacities below $1.5 \mathrm{MW}$.

There are not much researches in this subject. In one study, Tuma and Sekavcnik ${ }^{11}$ obtained enthalpy-entropy diagrams for expansion of natural gas of different composition for use in electrical power generation via expansion engines in CGSs.

Expansion engines are similar to reciprocating compressors in terms of geometry and simulation method. Hence, the researches on thermodynamic modeling of reciprocating compressor are also presented. The reciprocating compressors have been modeled with various methods. These methods could be generally classified into global models and differential models where the variable depends on crank angle ${ }^{12}$. Stouffs et al. ${ }^{12}$ presented a global model for the thermodynamic analysis of reciprocating compressors. Their model is based on five mains and four secondary dimensionless physically meaningful parameters and expressions for the volumetric effectiveness, the work per unit mass and the indicated efficiency were derived. Casting et al ${ }^{13}$ simulated compressor behavior using efficiency definitions such as volumetric, isentropic and effective. They supposed that these efficiencies depend essentially on two parameters, 
the dead volumetric ratio, having particular influence on volumetric efficiency, and a friction factor mainly influencing both isentropic and effective efficiencies. Elhaj et al. ${ }^{14}$ presented a numerical simulation of a two-stage reciprocating compressor for the development of diagnostic features for predictive condition monitoring. Winandy et al. ${ }^{15}$ presented a simplified model of an open-type reciprocating compressor. Their analysis revealed the main processes affected the refrigerant mass flow rate and the compressor power and the discharge temperature. Ndiaye et al. ${ }^{16}$ presented a dynamic model of a hermetic reciprocating compressor in on-off cycling operation. Farzaneh-Gord et al. ${ }^{17}$ investigated optimizing reciprocating air compressor design parameters thermodynamically. In this research, based on first law of thermodynamic, conservation of mass and valves modelling, in-cylinder pressure, in-cylinder temperature and valves motions at various crank angles was predicted.

The modifications of design parameters of such expansion engines lead to more efficient use of the machines. By modeling of these engines, it is possible to study effects of various parameters on their performance and to identify the optimum design parameters. The modeling and simulation could also enable us to diagnosis possible fault which degrade expansion engines performance. In this research, the main goal is to model one-sided reciprocating expansion engine thermodynamically for medium pressure range. The modeling is based on first law of thermodynamics, conversation of mass and ideal gas assumptions. Also, the working fluid is assumed as an ideal gas. The model could foretell in-cylinder pressure and in-cylinder temperature at various crank angles. Also, the effects of the engine geometrical characteristics, such as intake and exhaust port area and ports timing on the Indicated work per cycle output are studied.

\section{THE TOWN BORDER STATIONS (TBS)}

When a natural gas pipeline approaches a city, the high-pressure gas has to be reduced to a distribution level. A Town Border Station (TBS) is a pressure reducing point. Currently, the pressure reduction is carried out by throttling valve while considerable amount of pressure energy is wasted. One of the equipment which could be used to recover this waste energy is the reciprocating expansion engine. Inlet Gas has a high pressure $\left(\mathrm{P}_{\mathrm{NG}-1}\right)$ and temperature $\left(\mathrm{T}_{\mathrm{NG}-1}\right)$ which is typically related to the ambient temperature $\left(T_{a m}\right)$. A schematic diagram of a typical TBS equipped with an expansion engine is shown in Figure 1.

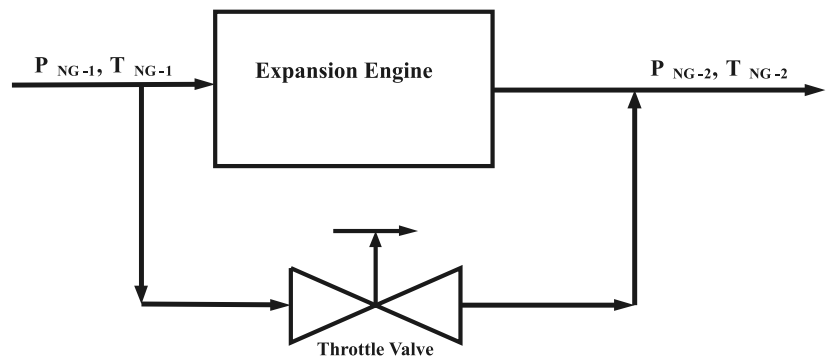

Figure 1. A Schematic diagram of TBS station equipped with an expansion engine

\section{CHEMICAL COMPOSITIONS OF NATURAL GAS}

Table 1 shows the composition of a type of natural gas which consumed in Iran based on the Khangiran refinery official website. By realizing that Methane is almost occupied the natural gas, here; it is assumed that Methane is the only substance in the Natural gas.

Table 1. Experimental analysis of natural gas composition the Khangiran refinery (the Khangiran refinery official website) $)^{18}$

\begin{tabular}{|l|c|c|}
\hline Component & Chemical formula & $\begin{array}{c}\text { Experimental Analysis } \\
\text { [mole Fraction \%] }\end{array}$ \\
\hline Carbon dioxide & $\mathrm{CO}_{2}$ & 0.055 \\
\hline Nitrogen & $\mathrm{N}_{2}$ & 0.428 \\
\hline Methane & $\mathrm{CH}_{4}$ & 98.640 \\
\hline Ethane & $\mathrm{C}_{2} \mathrm{H}_{6}$ & 0.593 \\
\hline Propane & $\mathrm{C}_{3} \mathrm{H}_{8}$ & 0.065 \\
\hline Iso butane & $\mathrm{C}_{4} \mathrm{H}_{10}$ & 0.015 \\
\hline n-Butane & $\mathrm{C}_{4} \mathrm{H}_{10}$ & 0.034 \\
\hline Iso-Pentane & $\mathrm{C}_{5} \mathrm{H}_{12}$ & 0.026 \\
\hline$+\mathrm{C}_{6}$ & $+\mathrm{C}_{6}$ & 0.125 \\
\hline & & Total $=100 \%$ \\
\hline
\end{tabular}

\section{THERMODYNAMIC ANALYSIS}

The schematic diagram of a reciprocating expansion engine with suction and discharge ports is shown in Figure 2. The rotary motion of crankshaft is converted to the reciprocating motion of piston by connecting rod. It is assumed that no leakage take place in the engine. Considering inside gas an open thermodynamic system, the governing equation for simulating the engine is presented in this section.

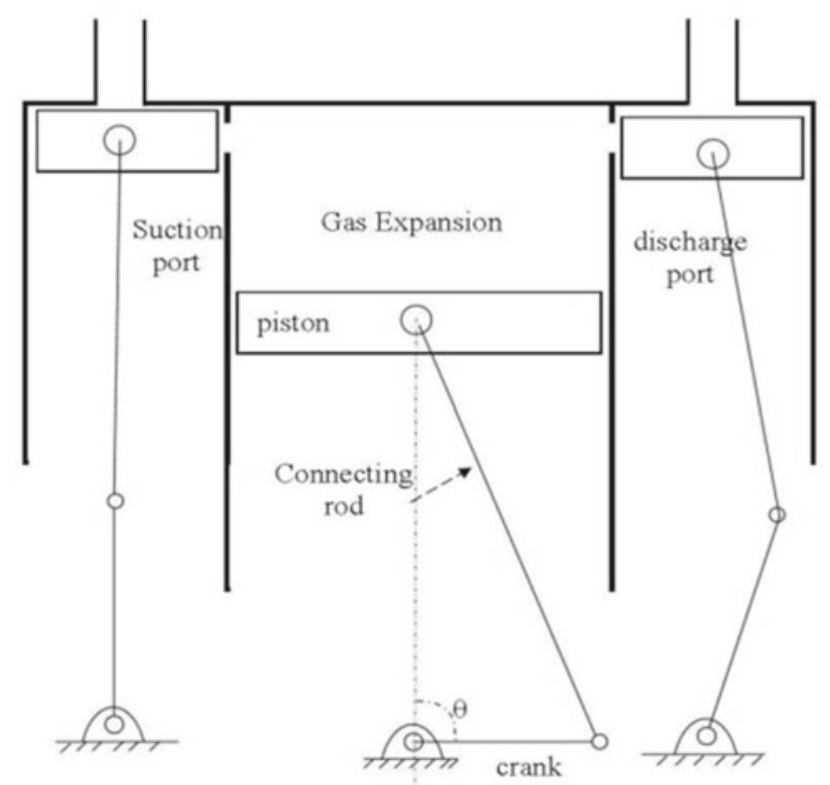

Figure 2. A schematic diagram of an Reciprocating Expansion Engine

\section{First Law Thermodynamics Equation}

To develop a numerical method, the continuity and first law of thermodynamics has been applied to the cylinder to find two thermodynamics properties. The cylinder wall, cylinder head and piston end face are considered as boundaries for control volume. The first law of thermodynamic is written as follow:

$\dot{Q}_{c v}+\sum \dot{m}_{i}\left(h_{i}+\frac{V_{i}^{2}}{2}+g z_{i}\right)=\sum \dot{m}_{e}\left(h_{e}+\frac{V_{e}^{2}}{2}+g z_{e}\right)+\frac{d}{d t}\left[m\left(u+\frac{V^{2}}{2}+g z\right)\right]_{c v}+\dot{W}_{c v}$ 
If variation kinetic and potential energies are neglected, then the first law of thermodynamic could be rewritten as follow:

$\frac{d Q_{\alpha}}{d t}+\frac{d m_{i}}{d t} h_{i}=\frac{d m_{e}}{d t} h_{e}+\frac{d}{d t}(m u)_{c}+\frac{d W_{w}}{d t}$

The work variation can be calculated as follow:

$\frac{d W_{c v}}{d t}=\mathrm{P}_{c v} \frac{d V_{c v}}{d t}$

Combining equation (2) and (3), the following equation could be easily driven:

$\frac{d Q_{c v}}{d t}+\frac{d m_{i}}{d t} h_{i}=\frac{d m_{e}}{d t} h_{e}+\frac{d}{d t}(m u)_{c}+\mathrm{P}_{c v} \frac{d V_{v}}{d t}$

On the other hand, differentiating respect to time could be converted to crank angle by considering the following equation ${ }^{19}$ :

$\frac{d}{d t}=\frac{d}{d \theta} \times \frac{d t}{d \theta}=\omega \frac{d}{d \theta}$

In which, $\omega$ is the rotational speed of the crank shaft. Finally the first law thermodynamic equation driven as below:

$\frac{d}{d \theta}(m u)_{c v}=\frac{d Q_{c v}}{d \theta}+\frac{d m_{i}}{d \theta} h_{i}-\frac{d m_{e}}{d \theta} h_{e}-\mathrm{P}_{c} \frac{d V_{c v}}{d \theta}$

\section{Piston Motion Equation}

The exact expression for the instantaneous position of the piston displacement from top dead center in terms of the crank angle may be given by ${ }^{\mathbf{1 9}}$ :

$x(\theta)=\frac{S}{2}\left[1-\cos \theta+\frac{L}{a}\left(1-\sqrt{\left(1-\left(\frac{a}{L} \sin \theta\right)^{2}\right.}\right)\right]$

Where $a$ and $L$ are crank and rod lengths. The instantaneously volume of cylinder is given by:

$V_{c}=A_{c} \times s(\theta)+V_{0}$

Where $V_{0}$ is the dead volume.

\section{Mass Conservation Equation}

Considering the in-cylinder gas as a control volume the continuity (conservation of mass) equation may be written as follows:

$\frac{d m_{c v}}{d \theta}=\frac{d m_{s}}{d \theta}-\frac{d m_{d}}{d \theta}$

Where $\frac{d m_{s}}{d \theta}$ and $\frac{d m_{d}}{d \theta}$ are the mass flow rates through suction and discharge ports respectively, which are calculating from following equations ${ }^{19}$ :

$\dot{m}_{s}=\left\{\begin{array}{lll}\rho_{s} A_{s} \sqrt{\left.\frac{2\left(P_{s}-P_{c}\right)}{\rho_{s}}\right)} \text { for } & P_{s}>P_{c} \text { and } x_{s}>0 \\ \rho_{c} A_{s} \sqrt{\left.\frac{2\left(P_{c}-P_{s}\right)}{\rho_{c}}\right)} \text { for } & P_{c}>P_{s} \text { and } x_{s}>0\end{array}\right.$

$\dot{m}_{d}=\left\{\begin{array}{lll}\rho_{c} A_{d} \sqrt{\left.\frac{2\left(P_{c}-P_{d}\right)}{\rho_{s}}\right)} \text { for } & P_{c}>P_{d} \text { and } x_{d}>0 \\ \rho_{d} A_{d} \sqrt{\left.\frac{2\left(P_{d}-P_{c}\right)}{\rho_{d}}\right)} \text { for } & P_{d}>P_{c} \text { and } x_{d}>0\end{array}\right.$

Where $A_{s}$ and $A_{d}$ are the flow areas through the suction and discharge valves which take place from cylinder respectively. Suction and discharge Ports are rotated with crank shaft force. Here, this motion is simulated as sinusoidal motion. They are given by:

$\mathrm{A}=\mathrm{A}_{\max } \sin \left(\frac{\theta-\theta_{\text {Open }}}{\theta_{\text {Close }}-\theta_{\text {Open }}} \pi\right)$
In which:

$A_{\max }=\left\{\begin{array}{l}A_{s(\max )}=2 \pi r_{s} \\ A_{d(\max )}=2 \pi r_{d}\end{array}\right.$

Where $r_{s}$ and $r_{d}$ are radiuses of suction and discharge valves respectively.

\section{Heat Transfer Equation}

Heat transfer could be calculated for each degree of crank angle from Equation (14) as:

$Q_{(\theta)}=U_{(\theta)} A_{c(\theta)}\left(T_{c(\theta)}-T_{a n}\right)$

Where $U_{(\theta)}, A_{c(\theta)}, T_{(\theta)}$ and $T_{a m}$ are the overall heat transfer coefficient, the heat transfer surface and in-cylinder gas temperature in each degree of crank angle and the ambient temperature respectively.

The overall heat transfer coefficient could be written as follow:

$\mathrm{U}=\frac{1}{\mathrm{~A}_{\text {Ref }} \sum R_{j}}$

In which $\sum R_{j}$ is the summation of heat resistants' of inside convection, wall conduction and outside convection that could be defined as ${ }^{19}$ :

$\sum R_{j}=\frac{1}{2 \pi r_{i} X_{(\theta)} h_{i}}+\frac{\log \frac{r_{o}}{r_{i}}}{2 \pi K_{i} X_{(\theta)}}+\frac{1}{2 \pi r_{o} X_{(\theta)} h_{o}}$

Where the outside convective heat transfer coefficient could be calculated as follow ${ }^{19}$ :

$N u=\frac{h_{o} D_{o}}{K_{\text {air }}}=\left(\frac{G r \times \operatorname{Pr}_{f}{ }^{2}}{2.435+4.884 \sqrt{\operatorname{Pr}_{f}}+4.953 \operatorname{Pr}_{f}}\right)^{0.25}$

Where $\operatorname{Pr}=\frac{C p \mu}{K}$ and $f$ index is shows properties in film temperature:

$T_{f}=\frac{T_{w}+T_{a n}}{2}$

The Grashof number is defined as:

$G r=\frac{\left(\frac{\pi D}{2}\right)^{3} g\left(\frac{T_{a m}-T_{w}}{T_{o}}\right)}{v^{2}}$

In which $T_{w}$ is cylinder wall temperature. Also the wall conduction heat coefficient can be assumed as conduction heat coefficient of carbon steel in ambient temperature that is assumed as $50 \mathrm{~W} / \mathrm{mK}$.

The Nusselt number of inside cylinder is calculated from Hassan's equation ${ }^{19}$ :

$N u=\frac{h_{i} D_{i}}{K_{\text {gas }}}=0.023 \mathrm{Re}^{0.8}$

where $\operatorname{Re}=\frac{C_{m} D}{v}$ is Reynolds number and $C_{m}=\frac{2 s N}{60}$ is mean piston speed with rotational speed of $N$.

\section{Ideal Gas Model}

Town border stations work in $0.4-1.7 \mathrm{MPa}$ pressure range. Figure 3 shows variation of $\mathrm{Z}$ factor in this pressure range in ambient temperature. Considering the Figure 3, ideal gas assumption is acceptable for methane in this range.

For the case of assuming ideal gas behaviour, the governing equation could be much simplified. Considering the following ideal gas assumptions:

$u=C_{v} T, h=C_{p} T, C_{p}-C_{v}=R, P V=m R T$ 


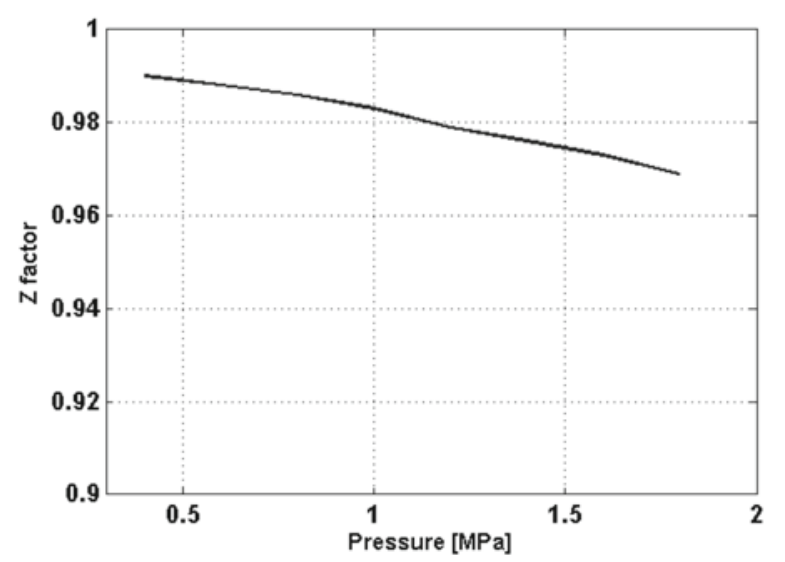

Figure 3. $\mathrm{Z}$ factor vs. pressure for methane in TBS pressure range

Consequently,

$\frac{d(m u)_{c v}}{d \theta}=m_{c v} C_{v} \frac{d T_{c v}}{d \theta}+T_{c v} C_{v} \frac{d m_{c v}}{d \theta}$

With replacing ideal gas assumptions and equation (14), the equation (6) could be simplified as below:

$m_{c v} C_{v} \frac{d T_{c v}}{d \theta}+T_{c v} C_{v} \frac{d m_{c v}}{d \theta}=U_{(\theta)} A_{c(\theta)}\left(T_{c(0)}-T_{a n}\right)+\dot{m}_{i} C_{p} T_{i}-\dot{m}_{e} C_{p} T_{e}-P_{c v} \frac{d V_{c v}}{d \theta}$

\section{Numerical Solution}

Differential equations that should be solved simultaneously are first law and continuity equations. With using Ideal gas model and discretization method, these equations could be written as below:

$\frac{T^{j+1}{ }_{c v}-T^{j}{ }_{c v}}{\Delta \theta}=\frac{1}{m_{c v}^{j}\left(C_{p}-R\right)}\left\{\begin{array}{l}U_{(\theta)}{ }^{j} A_{c v(\theta)}{ }^{j}\left(T_{c v}{ }^{j}-T_{a n}\right)+T_{i} C_{p} \dot{m}_{i}^{j}-P_{c v}{ }^{j}\left(\frac{V^{j+1}{ }_{c v}-V^{j}{ }_{c v}}{\Delta \theta}\right)- \\ T_{e} C_{p} \dot{m}_{e}^{j}-\omega C_{v}\left(\frac{m^{j+1}-m_{c v}^{j}{ }_{c v}}{\Delta \theta}\right) T^{j}{ }_{c v}\end{array}\right\}$

$\frac{m^{j+1}{ }_{c v}-m_{c v}^{j}}{\Lambda \theta}=\frac{\dot{m}_{i}^{j}-\dot{m}_{e}^{j}}{\Uparrow}$

Indicated work per In-cylinder mass is also calculated as following equation:

$\mathrm{W}_{\text {indicated }}=\frac{1}{\mathrm{~m}_{\mathrm{cv}}} \int \mathrm{PdV}=\frac{1}{\mathrm{~m}_{\mathrm{cv}}} \sum_{\mathrm{i} \neq}^{\mathrm{N}} \mathrm{P}_{\mathrm{i}} \mathrm{dV} \mathrm{i}_{\mathrm{i}}$

In which $\mathrm{N}$ is number of steps.

\section{RESULTS AND DISCUSSION}

In this study the results are presented for a reciprocating expansion engine with following specifications: $D_{i_{\text {cyld }}}=15 \mathrm{~cm}, D_{o_{\text {cyld }}}=18 \mathrm{~cm}, S=12 \mathrm{~cm}, r_{s}=2.5 \mathrm{~cm}, r_{d}=3 \mathrm{~cm}$, $T_{s}=280 \mathrm{~K}$

The pressure of discharge plenum and also pressure ratio are considered to be $0.4 \mathrm{MPa}$ and 4.25 , respectively. The effects of various parameters are also studied in separated sections.

Firstly, due to the lack of experimental results, for validation the mathematical method in this study, the results of numerical method have been compared with theoretical results. In the theoretical study, the suction and discharge valves assumed to be closed. Also the thermodynamic processes are considered to be isentropic. Based on these assumptions, the values of in-cylinder pressure could be calculated as follows:

$P_{c v}^{j+1}=P_{c v}^{j}\left(\frac{v^{j+1}}{v_{c v}^{j}}\right)^{k}$
Figure 4 compares the variation of in-control volume pressure between numerical values and theoretical results against cylinder volume. As shown this figure, there is a perfect match between the theoretical and numerical values.

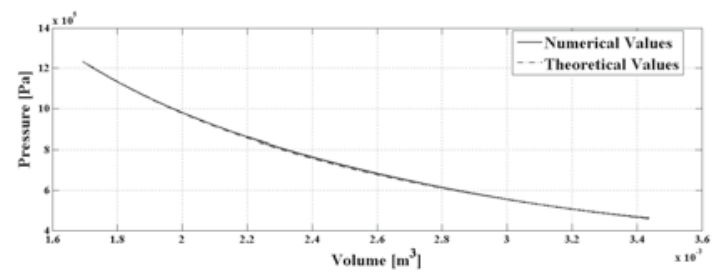

Figure 4. Comparison between numerical and theoretical values of in-cylinder pressure

The variation of in-cylinder pressure against crank angle is shown in Figure 5. In the numerical study, the suction and discharge pressure assumed constant.

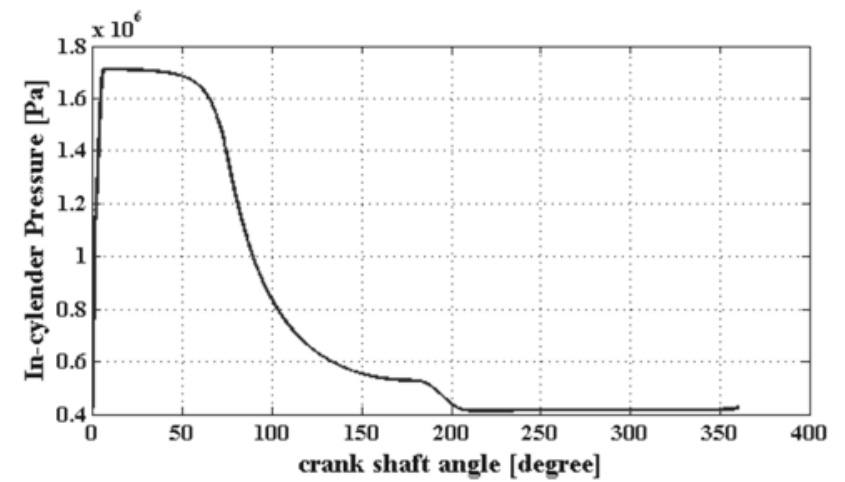

Figure 5. Variations of in-cylinder pressure against crank angle at $\omega=1000 \mathrm{rpm}$

Figure 6 illustrates variation of in-cylinder temperature in various crank angles. At the beginning, the temperature raises by suddenly entering large amount of mass into the system. Then start to decrease till half of the cycle. Decreasing of specific volume is the reason of the temperature dropping in suction process. In expansion process pressure and the temperature declining is occurred simultaneously. In the discharge process, constant pressure and declining density cause the temperature raising. It should be noted that, based on previous studies $^{\mathbf{2 0}, \mathbf{2 1}}$, the gas hydrate formation temperature for outlet pressure of TBS station, about $0.4 \mathrm{MPa}$, is around $-25 \mathrm{C}$. On the other hand, if the temperature is lower than the hydrate temperature, the natural gas flow must be preheated.

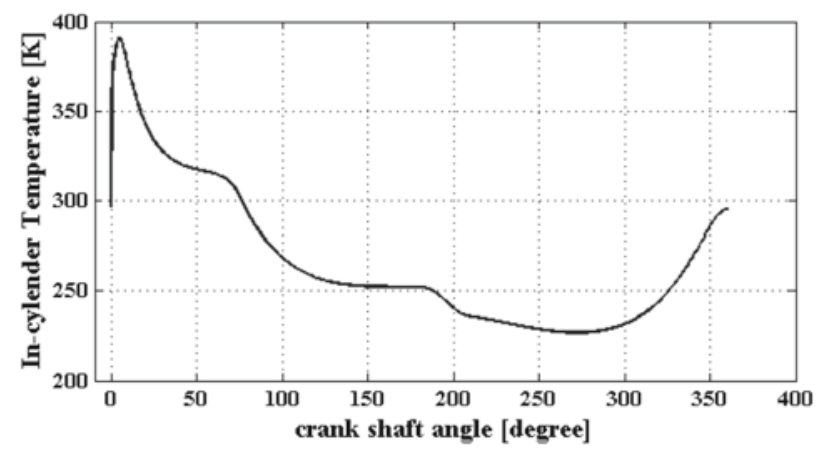

Figure 6. In-cylinder temperature variations vs. crank angle 
$\mathrm{P}-\mathrm{V}$ diagram of one cycle is shown in Figure 7. As expected suction and discharge processes are constant-pressure evolution. Expansion is a polytrophic evolution and compression of residual mass is a constant-volume process.

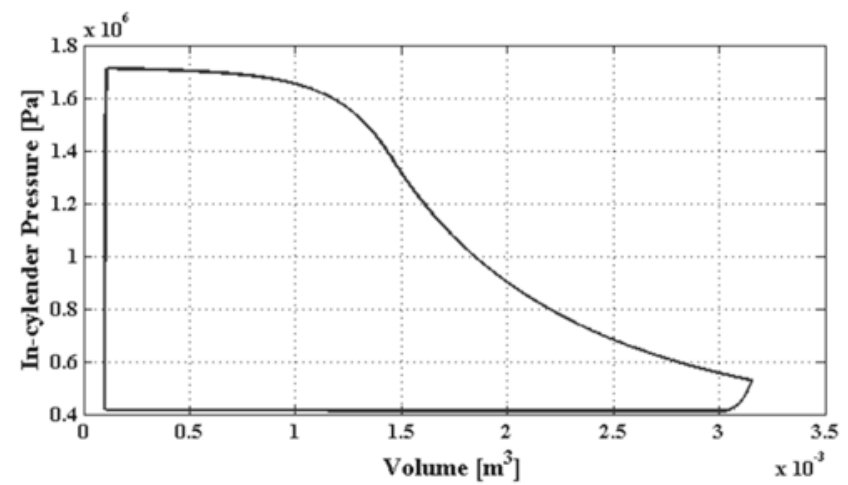

Figure 7. In-cylinder pressure variations vs. cylinder volume for one cycle

In Figure 8 the variation of in-cylinder gas density in one cycle is shown. Note from the figure, the variation of density in one cycle is similar with pressure variations.

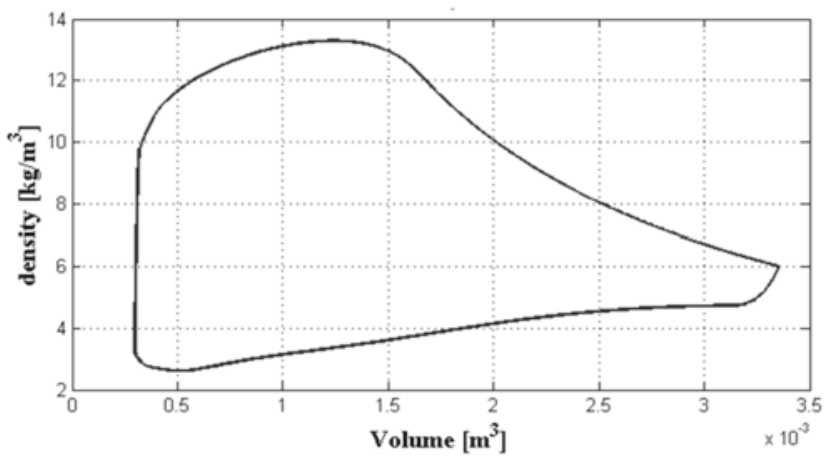

Figure 8. Variations of in-cylinder gas density vs. cylinder volume

The variations mass of in-cylinder gas is shown as Figure 9. The mass of in-cylinder gas grows up when suction port is open. Then stay on $0.021 \mathrm{~kg}$ during the expansion process. Sudden discharging is happened by opening discharge port at first and discharge continues with more balanced procedure.

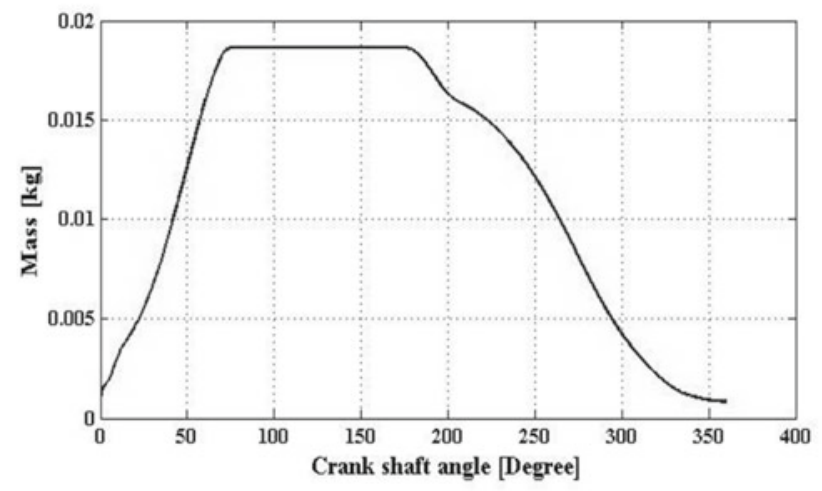

Figure 9. Variations of mass of in-cylinder gas vs. crank angle

\section{Effect of Port areas on engine performance}

The variation of Indicated work per cycle against different values of suction and discharge port is shown in
Figure 10. The considered suction port radius are 2, 2.5, $3,3.5$ and $4 \mathrm{~cm}$. and 2.5, 3, 3.5, $4 \mathrm{~cm}$ for discharge port. According to Figure 10 suction port is optimized at $\frac{R_{S}}{R_{\text {cyld }}}=0.28 \mathrm{~cm}$ of the most important variables of motor optimizing. What is certain is that by increasing the mass of the gas in cylinder, the amount of indicated work increases. So investigations are done on variation of Indicated work per cycle in mass unit of gas.

The work variation per mass of system against radius of suction port is shown in Figure 10. This graph which is plotted at different values of the radius of the discharge port provides the following important notes:

1 - optimized diameter of suction port is $\frac{R_{S}}{R_{c y l d}}=0.28 \mathrm{~cm}$.

2 - Although by increasing the diameter of the exhaust port the amount of Indicated work per cycle increases in effect of reducing gas pressure on the piston in the discharge phase, but Figure 11 indicates that the exhaust gas temperature is reduced in this case.

Figure 10 also shows that increasing exhaust port radius, increases the Indicated work per cycle. So the best discharge port diameter is the maximum possible diameter.

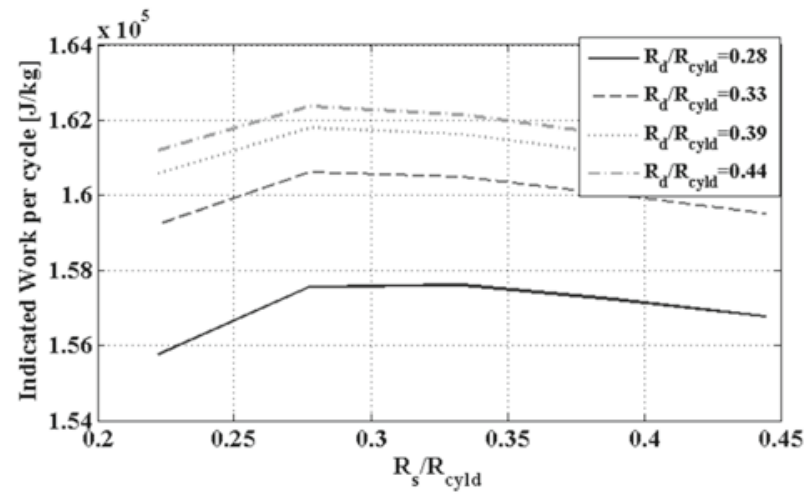

Figure 10. Variation of Indicated work per cycle vs. suction port radius in different discharge port radius

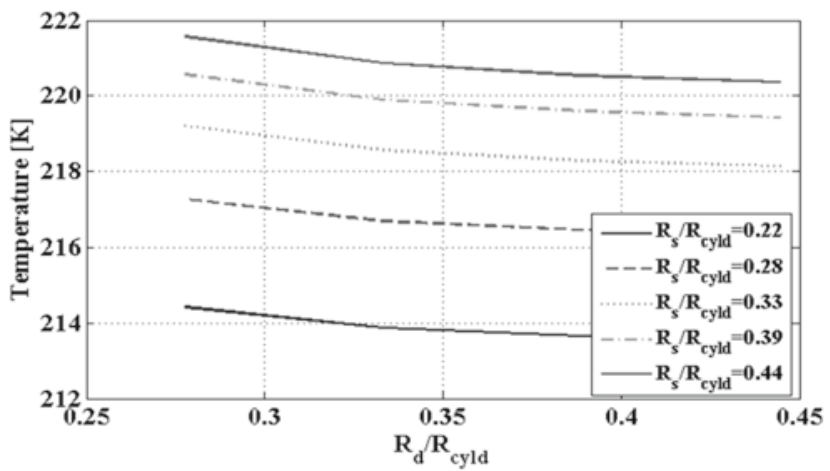

Figure 11. Variation of discharge temperature vs. discharge port radius in different suction port radius

\section{Effect of Port timing on engine performance}

For investigation of port timing it is assumed that suction port opens at the beginning of the cycle $(\theta=0)$ and discharge port closes at the end of the cycle. Accordingly, time of closure suction port and opening of discharge port are studied. 
According to port areas and motor geometry, the minimum adoptable value for suction port closure is 75 degree crank angle. Based on this, three amounts of 75,85 and 95 degrees for crank were tested for suction port closure. Discharge port opening is also considered in 170 to 190 crank angles.

The results of this study which is illustrated in Figure 12 shows that the highest mass unit Indicated work per cycle occurs on the angle of 75 degree for suction port closure. Figure 13 shows the effect of discharge port timing on Indicated work per cycle. It shows that degree 182 is the best time for discharge port opening.

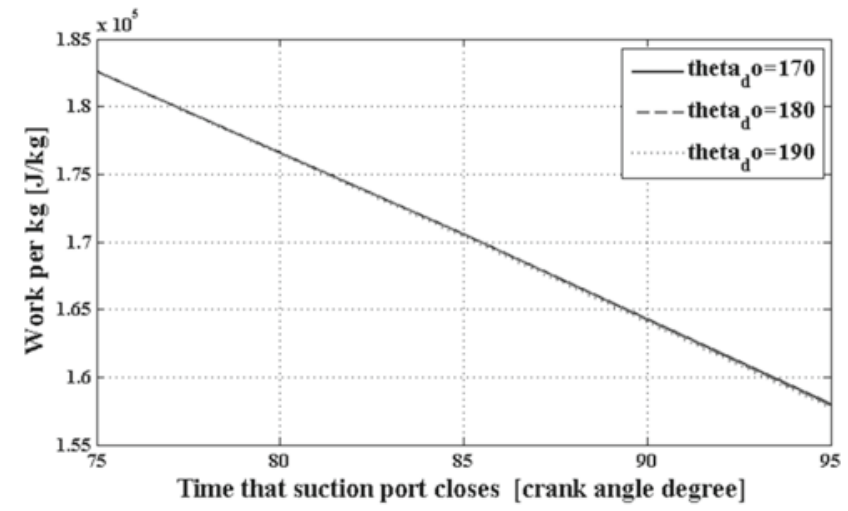

Figure 12. Variation of Indicated work per cycle vs. suction duration

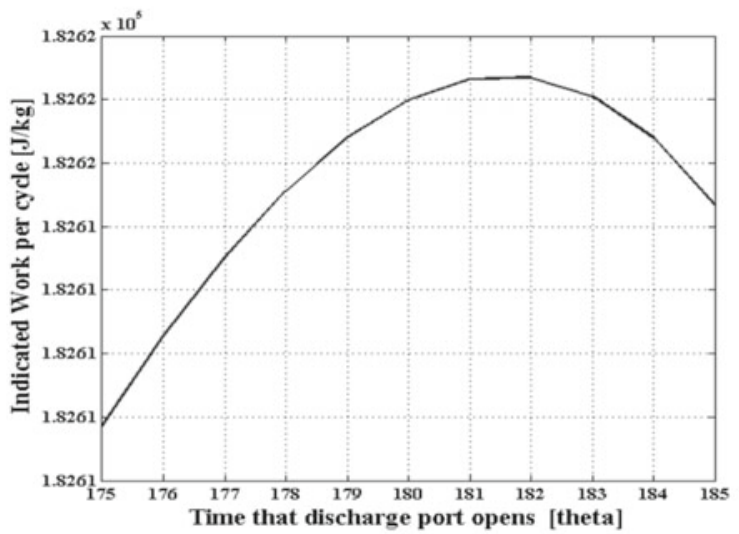

Figure 13. Variations of Indicated work per cycle vs. discharge duration

In addition to the amount of work, the average exit gas temperatures during discharge process are also investigated. Based on the results from Figure 14, delay in closing suction port and also delay in opening discharge port causes rise to outlet temperature.

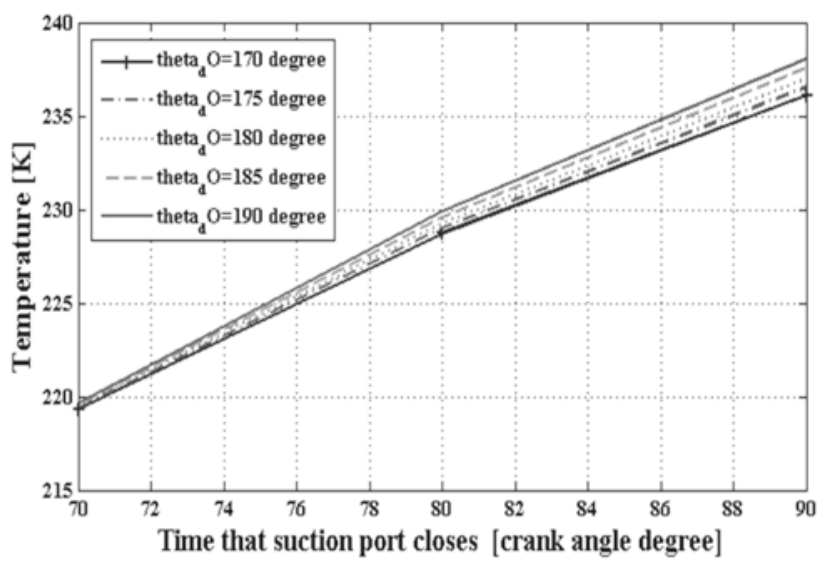

Figure 14. Variation of discharge temperature vs. discharge duration

\section{Cost analysis}

The cost analysis is carried out for the expansion engine installation. In this method, revenues are calculated as a result of electricity production in MWh per year. Based on result, the average power generation in TBS station with employing expansion engine is about $46.2 \mathrm{~kW}$. The net electricity production is around $368.064 \mathrm{MWh}$ in 360 days of the year. It should be noted that 5 days per year is considered for maintenance periods. The benefit of this electricity production is calculated to be 22083.84 US\$ based on the current electricity price in Iran which is 6 Cents $/ \mathrm{kWh}$.

Finally the payback ratio may be calculated as follows: PaybackRatio $=\frac{(\text { Capital }+ \text { O\& } M \text { Costs })}{\text { Benefit }}=\frac{(30000+5000)}{22083.84}=1.58$

Table 2 shows the detailed cost analysis for the expansion engine system. Based on these values, the payback period has been calculated to be around 1.58 years. This unveiled the cost effectiveness of the proposed system.

Table 2. The cost analysis of the expansion engine system

\begin{tabular}{|l|c|}
\hline $\begin{array}{l}\text { Total capital cost for expansion } \\
\text { engine installation }\end{array}$ & 30000 US\$ \\
\hline Annual O\&M costs & 5000 US\$ \\
\hline Cost of electricity per kWh & 0.06 US\$ \\
\hline Annual power generation & $368.064 \mathrm{MWh}$ \\
\hline Annual benefit & 22083.84 US\$ \\
\hline Payback period & 1.58 year \\
\hline
\end{tabular}

\section{CONCLUSIONS}

Expansion engines can be used widely in gas pressure reduction stations due to their ability to recover considerable amount of energy. Understanding the behavior of the reciprocating engines and studying effects of various parameters are interesting subjects. Expansion engines have many advantages over expansion turbines. The main advantages of these engines are capable of operating with wet natural gas flow (hydrate in natural gas). Also, expansion engines are less expensive than expansion turbines especially for capacities below 1.5 MW.The mathematical modeling is proved to be an effective tool to study performance of the expansion engines.

A mathematical model has been developed based on the conservation of mass and first law of thermodynamics to study the performance of these engines. The model could predict in-cylinder pressure, in-cylinder temperature and mass flow rates at various crank angles. The indicator work per unit mass of gas is also calculated. The effects of various parameters on the performance of the engine have been investigated.

The results show that ports timing is optimized at 75 degrees of crank angle for closing suction port and 182 degrees of crank angle for opening discharge port.

Variation of indicator work verses ratio of discharge to suction ports shows that there is a specific value (about 0.85 ) in which the indicator work per unit mass is maximized. This point could be treated as optimum design value for discharge to suction port area.

\section{NOMENCLATURE}

A area $\left(\mathrm{m}^{2}\right)$

a crank $(\mathrm{m})$ 


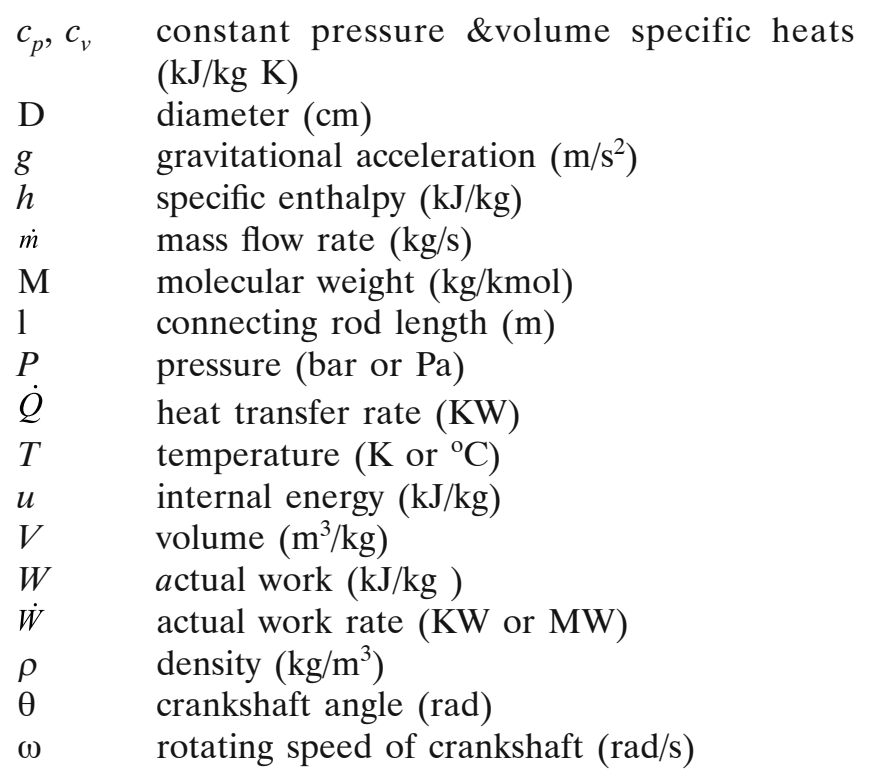

$\begin{array}{ll}\text { Subscript } \\ \text { amb } & \text { ambient } \\ \text { d } & \text { discharge condition } \\ \text { f } & \text { film } \\ \text { o } & \text { outside condition } \\ \text { cV } & \text { control volume condition } \\ \text { i } & \text { inside condition } \\ \text { s } & \text { suction condition } \\ \text { cyld } & \text { cylinder } \\ \text { W } & \text { wall }\end{array}$

\section{ACKNOWLEDGEMENT}

This study has been supported financially by Khorasan Razavi Gas Company.

\section{LITERATURE CITED}

1. Bisio, G., Thermodynamic Analysis of the Use of Pressure Exergy of Natural Gas, Energy (1995). Vol. 20. No. 2. pp. 161-167, DOI: 10.1016/0360-5442(94)00074-D.

2. Pozivil, J., (2004). Use of expansion turbine in natural gas pressure reduction stations. J. Acta Montanistica Slovaca 9, 258-260.

3. Greeff, I.L., Visser, J.A, Ptasinski, K.J. \& Janssen, F.J.J.G. (2004). Using turbine expanders to recover exothermic reaction heat-flow sheet development for typical chemical processes, Energy, Vol. 29, pp 2045-2060. DOI: 10.1016/j. energy.2004.03.048.

4. Mirandola, A., Minca, Energy Recovery by Expansion of High Pressure Natural Gas. 21st Intersociety Energy Conversion Engineering Conference. San Diego, California(Aug. 25-29, 1986).

5. Farzaneh-Gord, M. \& Magrebi, J. (2009). Exergy of natural gas flow in Iran's natural gas fields, Int. J. Exergy, 6, (1), 131-142, DOI: 10.1504/IJEX.2009.023349.

6. Farzaneh-Gord, M., Deymi Dasht-Bayaz, M., (2008). Recoverable energy in natural gas pressure drop stations: a case study of the Khangiran gas refinery. Energy Exploration \& Exploitation; 26, 71-82. DOI: 10.126/0144598087855260508.

7. Farzaneh-Gord, M., Hashemi, Sh. \& Sadi, M. (2009). Energy destruction in Iran's natural gas pipe line network, Energy Exploration and Exploitation, 25, (6) DOI: 10.1260/014459807783791809.

8. Farzaneh-Gord, M. \& Deymi Dasht-bayaz, M. (2009). A New Approach for Enhancing Performance of a Gas Turbine (case study: Khangiran Refinery), Applied Energy 86, 2750-2759, DOI: 10.1016/j.apenergy.2009.04.017.
9. Farzaneh-Gord, M. \& Kargaran, M. (2010). Recovering energy at entry of natural gas into customer premises by employing a Counter-Flow vortex tube, oil \& Gas Science and Technology-Revue de l'IFP, Vol. 65, No. 6, pp. DOI: 903-912, DOI: $10.2516 /$ ogst/2009074.

10. Farzaneh-Gord, M. \& Sadi, M., (2008). Enhancing Energy Output in Iran's Natural Gas Pressure Drop Stations by Cogeneration, Journal of energy Institute, Vol. 81, No 4, pp. 191-196. 11. Tuma, M. \& Sekavcnik, M. (1997). Power generation by natural gas expansion engines, Fuel and Energy Abstracts.

12. Stouffs, P., Tazerout, M. \& Wauters, P. (2000). Thermodynamic analysis of reciprocating compressors. Inter. J. Therm. Sci. 40, 52-66.

13. Castaing-Lasvignottes, J. \& Gibout, S., Dynamic simulation of reciprocating refrigeration compressors and experimental validation. Inter. J. Refriger. 33 (2010), 381-389, DOI: 10.1016/J. IJREFRIG.2009.10.007.

14. Elhaji, M., Gu, F., Ball, A.D., Albarbar, A., Al-Qattan, M. \& Naid, A. (2008). Numerical simulation and experimental study of a two-stage reciprocating compressor for condition monitoring. Mechanical Systems and Signal Processing, 22, 374-389 DOI: 10.1016/j.ymssp.2007.08.003.

15. Winandy, E., Saavedra, O. \& Lebrun, J., Simplified modeling of an open-type reciprocating compressor. Int. J. Sci. 41 (2002), 183-192. DOI: 10.1016/S1290-0729(01)01296-0.

16. Ndiaye, D. \& Bernier, M. (2010). Dynamic model of a hermetic reciprocating compressor in on-off cycling operation (Abbreviation: Compressor dynamic model). Appl. Therm. Engine. 30, 792-799, DOI: 10.1016/j.applthermaleng.2009.12.007. 17. Farzaneh Gord, M., Niazmand, A. \& Deymi-DashteBayaz, M. (2013). Optimizing reciprocating air compressor design parameters based on first law analysis, U.P.B. Sci. Bull., Series D, Vol. 75, 4, 2013.

18. Khangiran refinery official website, http://khangiran.com/ pages/Products.htm

19. Sukhyung, L. (1983). First law analysis of unsteady processes with application to a charging process and a reciprocating compressor. The Ohaio state University; A Thesis presented in Partial Fulfillment of the Requirements for Degree of master Science.

20. Bahadori, A. \& Vuthaluru, H.B. A novel correlation for estimation of hydrate forming condition of natural gases. J. Nat. Gas Chem. 18, (2009) 453-457. DOI: 10.1016/S10039953(08)60143-7.

21. Farzaneh-Gord, M., Rahbari, H.R., Bajelan, M. \& Pilehvari, L. Investigation of hydrate formation in natural gas flow through underground transmission pipeline, J. Nat. Gas Sci. Engine. 15 (2013) 27-37. DOI: 10.1016/j.jngse.2013.09.001. 